\title{
Deficiencia de agua en el suelo y fijación biológica de nitrógeno en el cultivo de fréjol
}

\author{
Phaseolus vulgaris $\mathbf{L}$
}

\author{
Soil water deficiency and biological Nitrogen fixation in common bean crop Phaseolus vulgaris $\mathbf{L}$ \\ Calvache-Ulloa Marcelo Enrique ${ }^{1,2 *}$, Córdova-Yanchapanta Vicente ${ }^{3}$, Cruz-Tobar Saúl Eduardo ${ }^{1}$
}

\begin{tabular}{|c|}
\hline Datos del Articulo \\
\hline $\begin{array}{l}{ }^{1} \text { Facultad de Ciencias Agropecuarias } \\
\text { Universidad Técnica de Ambato, Tungu- } \\
\text { rahua, Ecuador. } \\
\text { Casilla postal: } 18-01-334 \text {. } \\
\text { Telf: }(+593) \text { 032746151- } 0984388229 \text {. }\end{array}$ \\
\hline $\begin{array}{l}{ }^{2} \text { Universidad Central del Ecuador. Av. } \\
\text { Universitaria, Quito 170129, Ecuador. } \\
\text { Telf: +59396065 4066. } \\
\text { mcalvache20@gmail.com }\end{array}$ \\
\hline $\begin{array}{l}{ }^{3} \text { Universidad Técnica del Cotopaxi Av. } \\
\text { Simón Rodríguez s/n Barrio El Ejido } \\
\text { Sector San Felipe. Latacunga - Ecuador. } \\
\text { Telf: +59303 2252205/2252307/2252346. } \\
\text { vicente.cordova@utc.edu.ec }\end{array}$ \\
\hline *Dirección de contacto: \\
\hline $\begin{array}{l}\text { Eduardo Cruz-Tobar } \\
\text { Facultad de Ciencias Agropecuarias. } \\
\text { Universidad Técnica de Ambato. } \\
\text { Tungurahua, Ecuador. } \\
\text { Casilla postal: 18-01-334. } \\
\text { Telf: (+593) 032746151-0984388229. } \\
\text { E-mail: se.cruz@uta.edu.ec }\end{array}$ \\
\hline Palabras clave: \\
\hline $\begin{array}{l}\text { Riego, } \\
\text { stress hídrico, } \\
\text { fijación biológica de nitrógeno, } \\
\text { sonda de neutrones, } \\
\text { fréjol, } \\
\text { Phaseolus vulgaris, } \\
\text { N15, } \\
\text { técnicas isotópicas. }\end{array}$ \\
\hline
\end{tabular}

J Selva Andina Biosph. 2017; 5(2):94-106.

\section{Historial del artículo.}

Recibido junio, 2017.

Devuelto septiembre 2017

Aceptado septiembre, 2017.

Disponible en línea, noviembre 2017.

\section{Editado por: \\ Selva Andina \\ Research Society}

\section{Key words:}

Irrigation,

water stress,

biological nitrogen fixation,

neutron probe,

Phaseolus vulgaris.

\section{Resumen}

La identificación de los estadios del cultivo de fréjol (Phaseolus vulgaris L.) en los que la deficiencia de agua que disminuye la fijación biológica de nitrógeno FBN, se realizó en ensayos de campo en un suelo Typic Haplustoll, franco arenoso del Centro Experimental La Tola, Pichincha, Ecuador. La temperatura media anual de la localidad es de $15{ }^{\circ} \mathrm{C}$ y la humedad relativa de 74\%, a una altitud de 2465 metros sobre el nivel del mar (msnm). Se registraron $123 \mathrm{~mm}$ de precipitación durante el período del experimento (julio y noviembre de 1995). Los tratamientos consistieron en 6 regímenes de humedad $S_{1}=$ Riego normal durante todo el ciclo, $S_{2}=$ Riego deficitario durante todo el ciclo, $S_{3}=$ Stress hídrico durante la fase de crecimiento vegetativo (20-50 días después de la siembra dds), $S_{4}=$ Stress hídrico durante la floración (53-81 dds), $S_{5}=$ Stress hídrico durante el llenado de la vaina (82-110 dds) y $S_{6}=$ Stress hídrico durante la maduración (111-130 dds). La humedad del suelo fue medida con una Sonda de neutrones hasta $0.40 \mathrm{~m}$ de profundidad, $24 \mathrm{~h}$ antes y después del riego. La FBN fue estimada con la metodología de N-15. Los resultados indican que el Stress hídrico durante todo el ciclo del cultivo $\left(S_{2}\right)$, durante la floración $\left(S_{4}\right)$ y durante el llenado de vainas $\left(S_{5}\right)$ afectó significativamente la fijación biológica del nitrógeno en el cultivo de fréjol. La restricción de riego durante la fase de crecimiento vegetativo $\left(S_{3}\right)$ y la maduración del grano $\left(S_{6}\right)$ no redujeron significativamente la fijación biológica de nitrógeno, alcanzando valores del $38 \%$, estadísticamente similares al cultivo con un régimen de riego normal durante todo el ciclo $\left(S_{l}\right)$.

(C) 2017. Journal of the Selva Andina Biosph. Bolivia. Todos los derechos reservados.

Abstract

The identification of the stages of the cultivation of beans (Phaseolus vulgaris L.), in which water deficiency reduces the biological fixation of nitrogen BNF, took place in field trials in a soil Typic Haplustoll, loamy sand of La Tola Experimental Center, Pichincha, Ecuador. The average annual temperature in the location is $15^{\circ} \mathrm{C}$ with a relative humidity of $74 \%$, at an altitude of 2465 meters above the sea level. $123 \mathrm{~mm}$ of rainfall were recorded during the period of the experiment (July at November 1995). The moisture regime treatments were as follows: $S_{I}=$ normal irrigation throughout the cycle, $S_{2}=$ deficit irrigation throughout the cycle, $S_{3}=$ water stress during vegetative growth (20-50 days after planting dap), $S_{4}=$ water stress during flowering (53-81 dap), $S_{5}=$ water stress during pod filling (82-110 dds), and $S_{6}=$ water Stress during ripening (111-130 dap). The soil moisture was measured with a neutron probe up to $0.40 \mathrm{~m}$ depth, $24 \mathrm{~h}$ before and after watering. The BNF was estimated with the methodology of $\mathrm{N}-15$. The results indicate that the water stress during the entire cultivation $\left(S_{2}\right)$, during flowering $\left(S_{4}\right)$ and during the filling of pods $\left(S_{5}\right)$ significantly affected the biological nitrogen fixation in the cultivation of beans. The restriction of watering during the vegetative growth $\left(S_{3}\right)$ and the maturation of grain $\left(S_{6}\right)$ not significantly reduced biological nitrogen fixation, reaching values of $38 \%$, statistically similar to the cultivation with a normal irrigation throughout the cycle $\left(S_{l}\right)$ regime. 


\section{Introducción}

El fréjol (Phaseolus vulgaris L.) es uno de los alimentos más importantes de la dieta diaria en la mayoría de la población ecuatoriana, Sus granos contienen un alto porcentaje de proteína (20-28 \%) y aminoácidos esenciales CORPEI 2009). La productividad del fréjol en el Ecuador es baja, debido a diversos factores como la falta de agua en el suelo, la presencia de plagas y enfermedades y la deficiencia de nutrientes en los suelos Peralta et al. s.f..

Como respuesta al uso desmedido de los fertilizantes de síntesis química y a los problemas ambientales que causan, se estimula cada vez más, la implementación de una agricultura sostenible, que permita reducir el impacto ambiental de la producción de alimentos (Singh et al. 2011). En este contexto, se ha dado particular importancia al rol de los microorganismos benéficos (Tikhonovich \& Provorov 2011, Ribeiro et al. 2013). Al ser una leguminosa, el fréjol tiene la capacidad de asociarse con bacterias fijadoras de nitrógeno conocidas como rizobium (López-Guerrero et al. 2012, Rai et al. 2012). Estos microorganismos le proporcionan a la planta nitrógeno a partir de la fijación biológica del nitrógeno atmosférico (FBN). El establecimiento de esta simbiosis es un proceso complejo, que implica un intercambio coordinado de múltiples señales entre la planta huésped y su microsimbionte (Murray 2011). El efecto de la sequía en las leguminosas es más compleja debido a la simbiosis con el Rhizobium, disminuyendo el proceso de infección, el desarrollo de los nódulos, la actividad de la nitrogenasa y acelerando la senescencia de los nódulos, (Hungría \& Vargas, 2000, Ramos et al. 1999).

Los trabajos de campo reportados en la literatura que indiquen la influencia del stress hídrico $(\mathrm{SH})$ en la FBN en el cultivo de fréjol son escasos. Castella 95 nos et al. (1996), en México y aplicando la metodología de N-15 se evidenció una pequeña diferencia en la FBN cuando se sometió a deficiencia de agua en la época vegetativa. En Ecuador, Calvache et al. (1997) señalan que la deficiencia de agua en el suelo en la etapa de floración disminuye drásticamente la productividad y la FBN.

Actualmente el método para evaluar la FBN en condiciones de campo, es la dilución isotópica del $\mathrm{N}$ 15. Para cuantificar el porcentaje y la cantidad de nitrógeno fijado de la atmosfera es necesario para una planta control, que tenga el mismo patrón radicular y la misma curva de absorción de nitrógeno (Hardarson et al. 1988, Hardarson 1993, Calvache et al. 1997).

La determinación del efecto del SH sobre la FBN en los diferentes estadios del cultivo de fréjol es de vital importancia para un manejo sostenible del agua y el mantenimiento de la productividad. En este trabajo se pretende obtener información de la influencia de la deficiencia hídrica en algunas etapas del cultivo de fréjol en la FBN. El objetivo fue identificar los estadios del cultivo de fréjol que presenten una mayor sensibilidad a la deficiencia de agua que afecte a la FBN.

\section{Materiales y métodos}

La variedad de fréjol (Phaseolus vulgaris L.) utilizada en el experimento fue INIAP 411, proporcionada por el departamento de semillas de la Estación Santa Catalina, INIAP (Instituto Nacional Autónomo de Investigaciones Agropecuarias). El cultivo de se realizó en el Centro Experimental "La Tola", perteneciente a la Facultad de Ciencias Agrícolas de la Universidad Central del Ecuador, (FCA-UCE) 
parroquia Tumbaco, cantón Quito, provincia de Pichincha. Sus coordenadas son: $00^{\circ} 13^{\prime}$ de latitud Sur, $78^{\circ} 22^{\prime}$ de longitud Oeste a una altitud de 2465 msnm.

Las características climáticas del lugar experimental son: temperatura media anual $16.2{ }^{\circ} \mathrm{C}$, humedad relativa anual $76.0 \%$ y precipitación anual 491.6 $\mathrm{mm}$, de acuerdo a los registros diarios de la Estación Meteorológica de Primer Orden, para el año 1996 (INAHMI, La Tola, 1996). Durante la ejecución del experimento (julio y noviembre de 1995), se registraron $123 \mathrm{~mm}$ de precipitación. El suelo del sitio del ensayo presenta una textura franco arcilloso, con $45 \%$ de arena, $27 \%$ de limo y $28 \%$ de arcilla. La densidad aparente (Da) fue de $1.32 \mathrm{gcm}^{-3}$. El punto de marchitez permanente fue de $12 \%$ y la capacidad de campo se estableció en $32 \%$ de humedad volumétrica del suelo. La porosidad fue de 45.3\%. De acuerdo con Calvache et al. (1995) el suelo del área del ensayo presenta menos del $2 \%$ de percolación profunda, lo que se considera despreciable desde el punto de vista de la aplicación de riego. El área del estudio fue homogénea con una pendiente menor al $3 \%$.

Previo a la implementación del experimento, el suelo fue preparado manualmente, retirando malezas y materiales extraños. Las labores incluyeron desfonde, nivelación y surcado. La siembra se efectuó manualmente, a razón de dos semillas por golpe, con una densidad de 166000 plantas de fréjol ha ${ }^{-1}$. Las semillas fueron certificadas por INIAP. Se sembró trigo manualmente al voleo en el surco central, como planta control para la aplicación de la técnica de N-15. (Hardason 1993). La semilla igualmente fue certificada y proporcionada por INIAP.

Para marcar el suelo con N-15, en los dos cultivos se aplicaron $20 \mathrm{~kg} \mathrm{ha}^{-1}$ de Nitrógeno, suficiente para efectuar la detección, en cuatro fracciones (vegetación, floración, llenado de vaina y maduración). Se utilizó sulfato de amonio grado experimental, proporcionado por la Comisión Ecuatoriana de Energía Atómica, marcado con 5\% de átomos en exceso de ${ }^{15} \mathrm{~N}$ como fuente de $\mathrm{N}$ (Hardarson 1993).

El agua aprovechable fue de $20 \%$, en función de la que se calculó la fracción fácilmente aprovechable y la fracción de SH para las plantas. Se considera que el $50 \%$ del agua aprovechable es la más fácilmente disponible para las plantas y el restante es difícilmente disponible, produciéndose la condición de SH. La condición de deficiencia se dio a niveles inferiores a $21 \%\left(0.21 \mathrm{~cm}^{3} . \mathrm{cm}^{-3}\right)$ de humedad del suelo (Calvache et al. (1995).

Los análisis de nitrógeno total y N-15 se realizaron en los laboratorios de la Ex Comisión Ecuatoriana de Energía Atómica (CEEA). El factor en estudio lo constituyó la inducción de SH durante las diferentes fases de desarrollo del cultivo de fréjol., como se detalla en la tabla 1. Debido a que el cultivo de referencia debe ser sometido a las mismas condiciones de manejo que el cultivo fijador, el SH también fue inducido en el trigo variedad Napo, con los correspondientes tratamientos: $S_{l}$ sin $\mathrm{SH}$ (riego según necesidades), $S_{2} \mathrm{SH}$ durante todo el ciclo de cultivo ( $\sin$ riego), $S_{3} \mathrm{SH}$ durante la fase de crecimiento vegetativo (20-52 días despues de la siembra dds), $S_{4}$ SH durante el llenado de la vaina (82-109 dds), ), $S_{5}=$ Stress hídrico durante el llenado de la vaina (82110 dds) y $S_{6}$ SH en la maduración (110-130 dds). Con el fin de evitar la interferencia de la precipitación con los regímenes de $\mathrm{SH}$, se cubrieron los surcos con láminas de plástico transparente de $8 \mathrm{~mm}$ de espesor, asegurando que el agua lluvia escurriera fuera de la parcela durante la duración del experimento. La superficie efectiva del plástico fue de 9 $\mathrm{m}^{2}(3 \times 3 \mathrm{~m})$. La evapotranspiración potencial (ETP) se determinó con el tanque evaporímetro MC (Calvache et al. 1995) y la lámina de riego por surcos se calculó de acuerdo al procedimiento citado por Cal- 
vache (2013). Las lecturas de humedad se realizaron cada $24 \mathrm{~h}$ y el riego se aplicó cada tres días, manualmente. Los valores de humedad se registraron hasta los $0.40 \mathrm{~m}$ de profundidad, considerando horizontes de $0.10 \mathrm{~m}$. Las lecturas de humedad se realizaron con la sonda de moderación de neutrones (Bacchi et al. 2002).

La unidad experimental estuvo constituida por una parcela de $3.0 \mathrm{~m}$ de largo, por $3.0 \mathrm{~m}$ de ancho, la misma que tenía cinco surcos distanciados $0.6 \mathrm{~m}$ entre ellos. La superficie total de la unidad experi- mental fue de $9.0 \mathrm{~m}^{2}$. Cada parcela tuvo 120 plantas de fréjol. La distancia de siembra fue de $0.6 \mathrm{~m}$ entre surcos y 0.20 m entre sitios, sembrándose dos semillas por sitio (Figura 1). El cultivo de referencia (trigo) se sembró en el surco central al voleo (aproximadamente 200 plantas), para que las condiciones de manejo de los dos cultivos sean similares. La distancia entre parcelas y entre bloques fue de 1.5 $\mathrm{m}$, para evitar interferencia en la aplicación del riego. El muestreo se realizó al azar tomando dos plantas de la parcela total.

Figura 1 Distribución de la unidad experimental

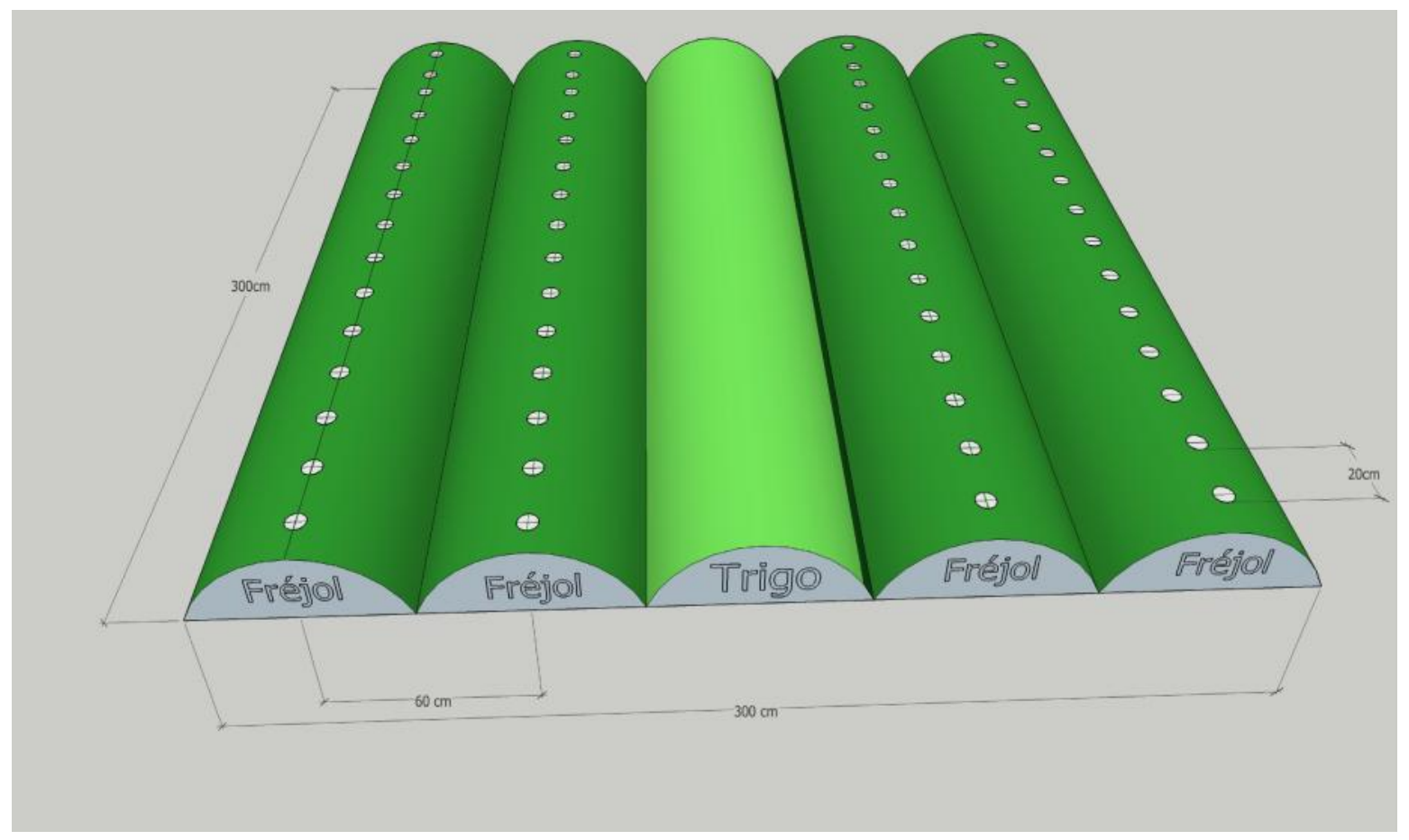

El ensayo de campo se manejó en base a un Diseño de Bloques Completos al Azar (DBCA) con seis tratamientos y cuatro repeticiones. Se calculó el análisis de varianza en función del diseño experimental planteado y se aplicó la prueba de rango múltiple de Duncan al 5\% para tratamientos para cada una de las fases de cultivo. Se establecieron las variaciones temporales aplicando ajustes de regresión.

Se registraron las siguientes variables, al final de cada una de las fases de cultivo establecidas, esto es a los 52, 81, 109 y 130 días, respectivamente:

Materia seca. (MS) Cuatro plantas completas tomadas al azar por parcela, se colocaron en una estufa a 
$60{ }^{\circ} \mathrm{C}$ por $72 \mathrm{~h}$, se determinó su peso seco y expresó en $\mathrm{kg} \mathrm{ha}^{-1}$.

Nitrógeno total. Se lo determinó mediante el método de Kjeldhal (Faust et al. 1987) y se reportó en kg $\mathrm{ha}^{-1}$.

Porcentaje de nitrógeno derivado de la atmósfera o fijación biológica de nitrógeno (FBN). Para determinar la proporción de $\mathrm{N}$ derivado de la atmósfera se aplicó la fórmula siguiente (Hardarson 1990):

Donde:

$$
\% \mathrm{NddA}=100-\% \mathrm{NddF}_{\text {fijador }}-\% \mathrm{NddS} \mathrm{S}_{\text {fijador }}
$$

$\%$ NddA $=$ Porcentaje de Nitrógeno derivado de la Atmósfera

$\% \mathrm{NddF}=$ Porcentaje de Nitrógeno derivado del Fertilizante

$\%$ NddS = Porcentaje de Nitrógeno derivado del Suelo

Los datos se analizaron mediante ANOVA, seguido de la nueva prueba de rango múltiple de Duncan para evaluar las diferencias de las medias (Duncan 1955).

\section{Resultados}

Tabla 2 Análisis de varianza para contenido de materia seca de la planta de fréjol

\begin{tabular}{ccccccc}
\hline Fuentes de & Grados de & & Cuadrados & medios & (fase) & \\
\hline variación & libertad & Vegetativa & Floración & Llenado & vaina & Maduración \\
Repeticiones & 3 & $1.95 \mathrm{NS}$ & $0.91 \mathrm{NS}$ & 2.35 & $\mathrm{NS}$ & $2.47 \mathrm{NS}$ \\
Tratamientos & 5 & $3.88 *$ & $9.79 * *$ & 6.8 & $* *$ & $15.71 * *$ \\
C.V. $(\%)$ & & 13.70 & 12.97 & 15.27 & & 6.80 \\
\hline NS $=$ No significativo $*$ = Significativo al $5 \% * * *=$ Significativo al $1 \%$ & &
\end{tabular}

Tabla 3 Prueba de Duncan al 5\% para tratamientos en la variable acumulación de materia seca en toda la planta $\left(\mathrm{kg} \mathrm{ha}^{-1}\right)$

\begin{tabular}{|c|c|c|c|c|c|c|c|c|c|c|c|}
\hline \multicolumn{12}{|c|}{ Promedios (fase) } \\
\hline \multicolumn{2}{|c|}{ Vegetativa } & \multicolumn{4}{|c|}{ Floración } & \multicolumn{3}{|c|}{ Llenado vaina } & \multicolumn{3}{|c|}{ Maduración } \\
\hline $\mathrm{S}_{1}$ & 548.70 & $a b$ & $\mathrm{~S}_{1}$ & 2501.40 & $\mathrm{a}$ & $\mathrm{S}_{1}$ & 4444.80 & $\mathrm{~b}$ & $\mathrm{~S}_{1}$ & 5361.30 & $\mathrm{~b}$ \\
\hline $\mathrm{S}_{2}$ & 412.80 & $\mathrm{c}$ & $\mathrm{S}_{2}$ & 1730.10 & $\mathrm{~b}$ & $\mathrm{~S}_{2}$ & 4252.80 & be & $\mathrm{S}_{2}$ & 5570.10 & $\mathrm{~b}$ \\
\hline $\mathrm{S}_{3}$ & 444.00 & be & $\mathrm{S}_{3}$ & 2583.60 & $\mathrm{a}$ & $\mathrm{S}_{3}$ & 6018.60 & $\mathrm{a}$ & $\mathrm{S}_{3}$ & 6963.00 & $\mathrm{a}$ \\
\hline $\mathrm{S}_{4}$ & 484.50 & abe & $\mathrm{S}_{4}$ & 1914.00 & $\mathrm{~b}$ & $\mathrm{~S}_{4}$ & 3867.00 & $\mathrm{c}$ & $\mathrm{S}_{4}$ & 4703.40 & $\mathrm{c}$ \\
\hline $\mathrm{S}_{5}$ & 520.80 & abe & $\mathrm{S}_{5}$ & 2809.80 & $\mathrm{a}$ & $\mathrm{S}_{5}$ & 5132.70 & $a b$ & $\mathrm{~S}_{5}$ & 5912.70 & $\mathrm{~b}$ \\
\hline $\mathrm{S}_{6}$ & 595.20 & $\mathrm{a}$ & $\mathrm{S}_{6}$ & 2953.20 & $\mathrm{a}$ & $\mathrm{S}_{6}$ & 6327.60 & $\mathrm{a}$ & $\mathrm{S}_{6}$ & 5323.80 & $\mathrm{~b}$ \\
\hline
\end{tabular}


Tabla 1 Requerimientos hídricos del cultivo de fréjol y láminas aplicadas

\begin{tabular}{|c|c|c|c|c|c|c|c|c|c|c|}
\hline \multirow[t]{2}{*}{ Días } & \multirow[t]{2}{*}{ Kc } & \multirow{2}{*}{$\begin{array}{r}\text { EMC } \\
\text { mm/frec } n\end{array}$} & ETr & \multicolumn{2}{|l|}{ Precip. } & \multicolumn{2}{|c|}{ Riego aplicado } & \multicolumn{2}{|c|}{ (mm/frecuencia) } & \multirow[b]{2}{*}{$\mathbf{S}_{6}$} \\
\hline & & & c mm/ f & $\mathrm{mm} /$ freo & $\mathbf{S}_{1}$ & $\mathbf{S}_{2}$ & $\mathbf{S}_{3}$ & $\mathbf{S}_{4}$ & $\mathrm{~S}_{5}$ & \\
\hline $6-9$ & 0.30 & 18.52 & 5.59 & 0.10 & 5.49 & 5.49 & 5.49 & 5.49 & 5.49 & 5.49 \\
\hline 12 & 0.30 & 16.96 & 5.09 & 0.00 & 5.09 & 5.09 & 5.09 & 5.09 & 5.09 & 5.09 \\
\hline 15 & 0.30 & 16.80 & 5.04 & 0.00 & 5.04 & 5.04 & 5.04 & 5.04 & 5.04 & 5.04 \\
\hline 18 & 0.30 & 14.24 & 4.27 & 0.00 & 4.27 & 4.27 & 4.27 & 4.27 & 4.27 & 4.27 \\
\hline 21 & 0.30 & 17.21 & 5.18 & 0.00 & 5.18 & & & 5.18 & 5.18 & 5.18 \\
\hline 24 & 0.32 & 13.50 & 4.51 & 0.50 & 4.01 & & & 4.01 & 4.01 & 4.01 \\
\hline 27 & 0.36 & 13.23 & 4.70 & 0.00 & 4.70 & & & 4.70 & 4.70 & 4.70 \\
\hline 30 & 0.40 & 14.15 & 5.64 & 0.00 & 5.64 & & & 5.64 & 5.64 & 5.64 \\
\hline 33 & 0.45 & 11.06 & 5.25 & 0.60 & 4.65 & & & 4.65 & 4.65 & 4.65 \\
\hline 36 & 0.51 & 7.41 & 3.96 & 0.40 & 3.56 & & & 3.56 & 3.56 & 3.56 \\
\hline 39 & 0.57 & 6.88 & 4.48 & 1.00 & 3.48 & & & 3.48 & 3.48 & 3.48 \\
\hline 42 & 0.63 & 7.65 & 4.97 & 0.20 & 4.77 & & & 4.77 & 4.77 & 4.77 \\
\hline 45 & 0.70 & 9.37 & 6.75 & 0.30 & 6.45 & & & 6.45 & 6.45 & 6.45 \\
\hline 48 & 0.76 & 10.13 & 9.25 & 2.00 & 7.25 & & & 7.25 & 7.25 & 7.25 \\
\hline 51 & 0.83 & 11.69 & 9.65 & 0.00 & 9.65 & & & 9.65 & 9.65 & 9.65 \\
\hline 54 & 0.89 & 3.40 & 5.23 & 2.50 & 2.73 & 2.69 & 2.73 & & 2.73 & 2.73 \\
\hline 57 & 0.94 & 7.32 & 10.28 & 3.60 & 6.68 & 2.23 & 6.68 & & 6.68 & 6.68 \\
\hline 60 & 0.99 & 8.99 & 10.01 & 2.90 & 8.01 & & 8.01 & & 8.01 & 8.01 \\
\hline 63 & 1.04 & 14.32 & 16.43 & 1.50 & 14.93 & & 14.93 & & 14.93 & 14.93 \\
\hline 66 & 1.08 & 13.25 & 16.44 & 2.00 & 14.44 & & 14.44 & & 14.44 & 14.44 \\
\hline 69 & 1.08 & 12.48 & 18.64 & 4.30 & 14.34 & & 14.34 & & 14.34 & 14.34 \\
\hline 72 & 1.14 & 10.47 & 11.90 & 4.10 & 7.80 & 1.30 & 7.80 & & 7.80 & 7.80 \\
\hline 75 & 1.15 & 14.38 & 16.60 & 6.30 & 10.30 & & 10.30 & & 10.30 & 10.30 \\
\hline 78 & 1.17 & 15.66 & 18.25 & 4.00 & 14.25 & & 14.25 & & 14.25 & 14.25 \\
\hline 81 & 1.17 & 12.38 & 14.47 & 5.00 & 9.47 & 1.36 & 9.47 & & 9.47 & 9.47 \\
\hline 84 & 1.16 & 11.18 & 13.01 & 7.00 & 6.01 & 2.55 & 6.01 & 6.01 & & 6.01 \\
\hline 87 & 1.15 & 14.73 & 16.98 & 10.00 & 6.98 & 0.22 & 6.98 & 6.98 & & 6.98 \\
\hline 90 & 1.13 & 15.53 & 17.62 & 5.10 & 12.52 & 0.19 & 12.52 & 12.52 & & 12.52 \\
\hline 93 & 1.11 & 11.08 & 12.30 & 6.80 & 5.50 & & 5.50 & 5.50 & & 5.50 \\
\hline 96 & 1.08 & 15.83 & 17.10 & 10.30 & 6.80 & 2.91 & 6.80 & 6.80 & & 6.80 \\
\hline 99 & 1.05 & 14.93 & 15.60 & 12.10 & 3.50 & 4.52 & 3.50 & 3.50 & & 3.50 \\
\hline 102 & 1.01 & 15.86 & 15.96 & 10.20 & 5.76 & 1.78 & 5.76 & 5.76 & & 5.76 \\
\hline 105 & 0.96 & 15.85 & 15.29 & 8.00 & 7.29 & & 7.29 & 7.29 & & 7.29 \\
\hline 108 & 0.92 & 9.75 & 8.98 & 4.00 & 4.98 & 1.92 & 4.98 & 4.98 & & 4.98 \\
\hline 111 & 0.88 & 11.34 & 10.83 & 1.00 & 9.83 & & 9.83 & 9.83 & 9.83 & \\
\hline 114 & 0.83 & 13.11 & 10.94 & 0.00 & 10.94 & 2.40 & 10.94 & 10.94 & 10.94 & \\
\hline 117 & 0.79 & 13.07 & 13.15 & 3.50 & 9.65 & 0.55 & 9.65 & 9.65 & 9.65 & \\
\hline 120 & 0.76 & 11.50 & 8.72 & 0.00 & 8.72 & 2.95 & 8.72 & 8.72 & 8.72 & \\
\hline 123 & 0.73 & 9.53 & 9.12 & 3.00 & 6.12 & 3.67 & 6.12 & 6.12 & 6.12 & \\
\hline 126 & 0.71 & 17.34 & 12.22 & 0.80 & 11.42 & 0.51 & 11.42 & 11.42 & 11.42 & \\
\hline 129 & 0.69 & 13.56 & 9.39 & 0.00 & 9.39 & & 9.39 & 9.39 & 9.39 & \\
\hline Total & & 544.12 & 430.69 & 123.10 & 307.59 & 51.65 & 248.24 & 204.64 & 248.25 & 241.52 \\
\hline
\end{tabular}


Tabla 4 Análisis de varianza para nitrógeno total

\begin{tabular}{ccccccc}
\hline Fuentes de & Grados de & \multicolumn{5}{c}{ Cuadrados medios (fase) } \\
\hline variación & libertad & Vegetativa & Floración & Llenado vaina & Maduración \\
\hline Repeticiones & 3 & $1.09 \mathrm{NS}$ & $1.82 \mathrm{NS}$ & 1.58 & $\mathrm{NS}$ & $1.54 \mathrm{NS}$ \\
Tratamientos & 5 & $3.79 *$ & $7.89 * *$ & 5.82 & $* *$ & $23.72 * *$ \\
C.V.(\%) & & 16.55 & 14.35 & 14.05 & 5.97 \\
\hline
\end{tabular}

NS = No significativo; $*$ = Significativo al $5 \% ; * *=$ Significativo al $1 \%$

Tabla 5 Prueba de Duncan al $5 \%$ para tratamientos en la variable nitrógeno total $\left(\mathrm{kg} \mathrm{ha}^{-1}\right)$

\begin{tabular}{|c|c|c|c|c|c|c|c|c|c|}
\hline \multicolumn{10}{|c|}{ Promedios (fase) } \\
\hline \multicolumn{3}{|c|}{ Vegetativa } & \multicolumn{2}{|c|}{ Floración } & \multicolumn{2}{|c|}{ Llenado vaina } & \multicolumn{3}{|c|}{ Maduración } \\
\hline$S_{1}$ & 23.62 & $\mathrm{a}$ & $\mathrm{S}_{1}$ & $77.13 \mathrm{a}$ & $\mathrm{S}_{1}$ & 132.21 & $a b$ & $\mathrm{~S}_{1}$ & $134.24 \mathrm{c}$ \\
\hline $\mathrm{S}_{2}$ & 16.21 & $\mathrm{c}$ & $\mathrm{S}_{2}$ & $50.35 \mathrm{~b}$ & $\mathrm{~S}_{2}$ & 118.40 & $\mathrm{~b}$ & $\mathrm{~S}_{2}$ & $118.43 \mathrm{~d}$ \\
\hline $\mathrm{S}_{3}$ & 17.41 & be & $\mathrm{S}_{3}$ & $81.44 \mathrm{a}$ & $\mathrm{S}_{3}$ & 160.17 & $\mathrm{a}$ & $\mathrm{S}_{3}$ & $179.95 \mathrm{a}$ \\
\hline $\mathrm{S}_{4}$ & 19.00 & abe & $\mathrm{S}_{4}$ & $58.15 \mathrm{~b}$ & $\mathrm{~S}_{4}$ & 108.99 & $\mathrm{~b}$ & $\mathrm{~S}_{4}$ & $136.86 \mathrm{c}$ \\
\hline $\mathrm{S}_{5}$ & 21.81 & $a b$ & $\mathrm{~S}_{5}$ & $79.13 \mathrm{a}$ & $\mathrm{S}_{5}$ & 116.81 & $\mathrm{~b}$ & $\mathrm{~S}_{5}$ & $155.95 \mathrm{~b}$ \\
\hline
\end{tabular}

Tabla 6 Análisis de varianza para porcentaje de fijación biológica de nitrógeno

\begin{tabular}{cccccccccc}
\hline Fuentes de & Grados de & \multicolumn{9}{c}{ Cuadrados medios (fase) } \\
\cline { 1 - 5 } variación & libertad & \multicolumn{2}{c}{ Vegetativa } & \multicolumn{2}{c}{ Floración } & \multicolumn{2}{c}{ Llenado vaina } & \multicolumn{2}{c}{ Maduración } \\
\hline Repeticiones & 3 & 0.28 & NS & 0.28 & NS & 0.37 & NS & 0.13 & NS \\
Tratamientos & 5 & 2.69 & NS & 2.33 & NS & 13.32 & $* *$ & 5.18 & $* *$ \\
C.V.(\%) & & 30.89 & & 34.62 & & 18.40 & & 32.72 \\
\hline
\end{tabular}

NS = No significativo; $* *=$ Significativo al $1 \%$

Tabla 7 Prueba de Duncan al $5 \%$ para tratamientos en la variable porcentaje de fijación biológica de nitrógeno

\begin{tabular}{|c|c|c|c|c|c|c|c|c|}
\hline \multicolumn{9}{|c|}{ Medias (fase) } \\
\hline & Vegetativa & & Floración & & nado & vaina & & Maduración \\
\hline $\mathrm{S}_{1}$ & 29.36 & $\mathrm{~S}_{1}$ & 27.66 & $\mathrm{~S}_{1}$ & 29.73 & $a b$ & $\mathrm{~S}_{1}$ & $36.47 \mathrm{a}$ \\
\hline $\mathrm{S}_{2}$ & 39.08 & $\mathrm{~S}_{2}$ & 17.24 & $\mathrm{~S}_{2}$ & 12. 83 & $\mathrm{~d}$ & $\mathrm{~S}_{2}$ & $16.95 \mathrm{~b}$ \\
\hline $\mathrm{S}_{3}$ & 23.02 & $\mathrm{~S}_{3}$ & 32.46 & $\mathrm{~S}_{3}$ & 30.65 & $a b$ & $\mathrm{~S}_{3}$ & $38.70 \mathrm{a}$ \\
\hline $\mathrm{S}_{4}$ & 29.58 & $\mathrm{~S}_{4}$ & 24.96 & $\mathrm{~S}_{4}$ & 25.47 & be & $\mathrm{S}_{4}$ & $21.75 \mathrm{~b}$ \\
\hline $\mathrm{S}_{5}$ & 20.35 & $\mathrm{~S}_{5}$ & 30.22 & $\mathrm{~S}_{5}$ & 18.58 & $\mathrm{~cd}$ & $\mathrm{~S}_{5}$ & $17.98 \mathrm{~b}$ \\
\hline $\mathrm{S}_{6}$ & 22.38 & $\mathrm{~S}_{6}$ & 39.92 & $\mathrm{~S}_{6}$ & 36.28 & $\mathrm{a}$ & $\mathrm{S}_{6}$ & $38.64 \mathrm{a}$ \\
\hline
\end{tabular}

\section{Discusión}

Inducción del stress hídrico. En función de las características hídricas del suelo de ensayo y de la curva de coeficiente de cultivo $(\mathrm{Kc})$ determinada por Calvache et al. (1997) para el cultivo de fréjol en condiciones locales, se establecieron los requerimientos de riego para cada uno de los tratamientos, el que se suspendió en las etapas indicadas para la inducción del SH. En el caso de la inducción de SH durante todo el ciclo de cultivo, los niveles de humedad del suelo descendieron a valores críticos cercanos al punto de marchitez permanente, por lo que se aplicaron cantidades de riego suficientes para elevar el contenido de humedad valores cercanos al techo de la condición de stress (Tabla 1, Figura 2). En la Figura 2 se muestran los valores de humedad registradas durante el ensayo y que validan los tra- 
tamientos aplicados, considerándose que la humedad entre 0.21 y $0.32 \mathrm{~cm}^{3} / \mathrm{cm}^{3}$ está en el rango de agua fácilmente aprovechable por la planta. El SH aplicado es efectivo en valores de humedad del suelo de 0.14 a $0.21 \mathrm{~cm}^{3} / \mathrm{cm}^{3}$. Se observa que la reducción de la humedad del suelo a niveles de stress ocurre en las $72 \mathrm{~h}$ de iniciada la restricción de riego en tanto que la recuperación de humedad es mucho más rápida, dependiendo de la velocidad de infiltración de humedad.

Materia seca de la planta de fréjol. Durante la fase vegetativa solamente los tratamientos $\mathrm{S}_{2}$ (stress en todo el ciclo) y $\mathrm{S}_{3}$ (stress durante la fase vegetativa), registraron una significativa disminución $(\mathrm{P}<0.05)$ de la cantidad de materia seca, con 412.80 y 444.0 $\mathrm{kg} \mathrm{ha}^{-1}$. En los restantes tratamientos los promedios son estadísticamente similares, probablemente debido a que el adecuado suministro de humedad asegura un buen desarrollo vegetativo en esta fase. Es evidente el efecto por falta de agua en la síntesis de sustancias que forman la materia seca, al restringirse severamente el crecimiento de la planta, reflejada en el campo como altura de planta y volumen foliar menores. La deficiencia de humedad en el suelo produce primariamente una reducción de la transpiración, lo que directamente incide en la cantidad de materia seca y biomasa sintetizada y acumulada en la planta (Fereres et al. 1992, Calvache et al. 1997 Marino et al. 2006), especialmente cuando la deficiencia de humedad se extiende por períodos prolongados.

El SH provocado en la fase de floración $\left(S_{4}\right)$ y el tratamiento de stress total $\left(S_{2}\right)$, con promedios de 1914.0 y $1730.10 \mathrm{~kg} \mathrm{ha}^{-1}$, respectivamente, resultó en un efecto reductor sobre la cantidad de biomasa acumulada. A pesar de que gran parte de la biomasa ya ha sido sintetizada durante la fase vegetativa, por los valores de materia seca obtenidos, se aprecia que 101 la planta aún no alcanzó su tamaño total, por lo que el crecimiento de la planta continúa aún durante la floración. El tratamiento $S_{3}$ (stress durante la fase vegetativa), muestra una recuperación significativa de su capacidad de crecimiento, logrando un promedio estadísticamente similar a los tratamientos que no han sufrido restricción de humedad (2583.60 $\left.\mathrm{kg} \mathrm{ha}^{-1}\right)$. La alta relación de metabolismo que tiene lugar durante la fase de floración, se fundamenta en que las células mesofílicas de las hojas requieren de un medio altamente hidratado para que una mayor cantidad de $\mathrm{CO}_{2}$ sea intercambiado entre la hoja y la atmósfera circundante, por lo tanto, si el suministro de agua es adecuado, se incrementa la asimilación de $\mathrm{CO}_{2}$ y la acumulación de materia seca (Campbell \& Stockle 1993, Marino et al. 2006).

Es evidente que la deficiencia de agua en el suelo en la fase de floración es la que provoca mayor daño a la planta, ya que no presenta la misma capacidad de recuperación en la siguiente fase, según se aprecia en la evaluación al final de la fase de llenado de vaina. Así, el tratamiento $S_{4}$ (SH durante la fase de floración) es el que menor cantidad de materia seca registra, con $3867.0 \mathrm{~kg} \mathrm{ha}^{-1}$. La sequía constante inducida durante todo el ciclo de cultivo $\left(S_{2}\right)$ también presenta una reducida acumulación de materia seca.

El déficit de agua durante la fase de maduración de vaina no parece afectar significativamente a la acumulación de materia seca, ya que se mantiene una distribución estadística de los tratamientos similar a la observada en la fase de llenado de vaina, siendo evidente aún hasta esta etapa que la deficiencia de humedad durante la floración limita en mayor proporción la síntesis y acumulación de materia seca. Durante la maduración de grano, si bien la actividad metabólica continúa, ésta lo hace en proporciones decrecientes, por lo que la planta entera se lignifica 
y pierde la capacidad de transpiración, principalmente en las vainas y granos. El SH en esta fase $\left(S_{6}\right)$, ocasiona la disminución de la cantidad de materia seca principalmente por granos secados prematuramente (Castellanos et al. 1996).

Nitrógeno total. La diferencia de acumulación de $\mathrm{N}$ para tratamientos es significativa $(\mathrm{P}<0.05)$ en la fase vegetativa y altamente significativa $(\mathrm{P}<0.01)$ en las restantes fases. La interdependencia entre la cantidad de materia seca asimilada y el nitrógeno total determinan un comportamiento estadístico similar, ya que a pesar de que el $\mathrm{N}$ es un factor limitante en el desarrollo del cultivo, la producción de materia seca puede ser positivamente correlacionada con la cantidad de $\mathrm{N}$ fijado (Hardarson et al. 1990).

Los tratamientos que recibieron adecuada cantidad de agua durante la fase vegetativa asimilaron mayor cantidad de N, así, $S_{1}, S_{4}, S_{5}$ y $S_{6}$ presentan promedios estadísticamente similares con valores entre 19.00 y $23.97 \mathrm{~kg} \mathrm{ha}^{-1}$. Es evidente el efecto de la deficiencia de humedad del suelo en el desarrollo vegetativo de la planta, en los tratamientos $S_{2}$ (16.21 $\mathrm{kg} \mathrm{ha}^{-1}$ ) y $S_{3}\left(17.41 \mathrm{~kg} \mathrm{ha}^{-1}\right)$, con stress durante todo el ciclo y durante la fase vegetativa, respectivamente. De acuerdo con el PPI (1988), la cantidad de N asimilada aumenta con la aplicación de niveles adecuados de humedad al suelo, lo que se traduce en un rendimiento mayor, pudiendo inclusive duplicar la producción si el suministro de agua se incrementa de niveles del $40 \%$ hasta el $90 \%$ de la capacidad de campo; esto es particularmente evidente en caso de cereales (maíz y sorgo). La cantidad de $\mathrm{N}$ asimilada por la planta en la fase de floración se vio significativamente reducida en el caso de deficiencia de humedad del suelo durante todo el ciclo de cultivo $\left(S_{2}\right)$ así como para la inducción de stress durante la fase de floración $\left(S_{4}\right)$. La fase más crítica en cuanto al suministro de agua es la de floración, ya que el metabolismo de la planta se ve totalmente deteriorado, sin indicios de recuperación, evidenciándose este efecto adverso aún en las evaluaciones en la fase de llenado de vaina y maduración de grano. La inducción de stress en las fases de llenado de vaina y maduración de grano, produce una reducción significativa en la cantidad de $\mathrm{N}$ total asimilado durante estas fases, especialmente en la de maduración de grano, en donde ocurrió la pérdida de hojas por maduración prematura y como efecto directo de la falta de agua (Castellanos et al. 1996).

En el caso de $S_{l}(\sin \mathrm{SH})$, la menor cantidad de N acumulado hasta la maduración probablemente se debe a que el suelo presentó humedad cercana al punto de capacidad de campo, reduciendo la disponibilidad de aire en los poros del suelo como lo indica la Figura 2, especialmente en el estrato de suelo de $0.30 \mathrm{~m}$; esto puede ser consecuencia de la anoxia intermitente provocada por la saturación del suelo, a pesar que de acuerdo con Wilkins (1987), las plantas que permanecen bajo condiciones de alta humedad del suelo tienden a adaptarse. Los restantes tratamientos y en especial el $S_{3}$ (stress en la floración) muestran un incremento sostenido en la acumulación de $\mathrm{N}$ hasta la maduración del grano.

Porcentaje de nitrógeno derivado de la atmósfera o fijación biológica de nitrógeno. Se registraron diferencias estadística $(\mathrm{P}<0.05)$ para el porcentaje de FBN entre los tratamientos solamente a partir de la fase de llenado de la vaina (tabla 6).

La FBN es estadísticamente similar para todos los tratamientos en los estadios iniciales de desarrollo de la planta, es decir, en la fase vegetativa y en la de floración, con promedios de hasta $39.92 \%$; la similitud estadística se debe a que en estas fases ocurre los procesos de formación de nódulos y la iniciación de la fijación propiamente dicha. A partir de la fase de floración se aprecia que las condiciones de humedad adecuada en el suelo permiten una mayor fijación de $\mathrm{N}$ atmosférico, por lo que los tratamien- 
tos sin stress hídrico en esta fase $\left(S_{1}, S_{3}, S_{6}\right)$ registraron los más altos promedios, con valores de 29.73 a $36.28 \%$; los niveles de agua entre el 60 y $70 \%$ de la capacidad de campo promueven condiciones óptimas para la ocurrencia de la infección rizobial, formación de los nódulos y el proceso mismo de la fijación de $\mathrm{N}$ atmosférico, ya que incrementa la cantidad y longevidad de los nódulos (Ramos et al. 1999).

El porcentaje de fijación de $\mathrm{N}$ atmosférico se redujo a $12.83 \%$ en la fase de llenado de vaina y a $16.95 \%$ en la fase de maduración para el caso de stress constante durante todo el ciclo de cultivo $\left(S_{2}\right)$, lo que se debe básicamente a la falla en los procesos de infección y formación de los nódulos en las etapas tempranas de desarrollo del cultivo (infectividad), e impidiendo un funcionamiento adecuado de un alto número de nódulos formados (efectividad), de acuerdo con Ramos et al. (1999), la inducción de SH por periodos superiores a los 44 días, causa una reducción de la actividad de la nitrogenasa a cero, pudiendo ocurrir daños estructurales irreversibles en los nódulos formados. Esto es evidente en el caso de la aplicación de $\mathrm{SH}$ en la fase de llenado de vaina $\left(S_{5}\right)$, el cual registra un $30.22 \%$ de FBN en la fase de floración, la cual se reduce hasta $18.58 \%$ cuando se aplica el stress, en este caso, la razón principal para la reducción de la FBN es la disminución del contenido de leghemoglobina lo que a su vez reduce significativamente el transporte activo de oxígeno dentro del bacteroide, imposibilitando la manutención de la simbiosis (Rathore et al. 1981). La aplicación de SH no parece ser lesiva a la FBN en la fase de maduración de los granos, ya que el tratamiento correspondiente presenta un alto porcentaje de fijación (38.64\%), Las diferencias estadísticas registradas en esta fase se deben al efecto residual de la aplicación de stress en las fases anteriores y a 103 que en algunos casos no fue evidente la recuperación de la actividad de los nódulos.

$\mathrm{Al}$ analizar la variación de los tratamientos detallada en la Figura 3, la fijación óptima se registró para la aplicación de stress en la fase de maduración del grano $\left(S_{6}\right)$, con una tendencia sigmoidea, la que señala a la fase de floración como la de mayor tasa de fijación de $\mathrm{N}$ atmosférico, también la irrigación de acuerdo a los requerimientos durante todo el ciclo de cultivo tiende linealmente a incrementar el porcentaje de $\mathrm{N}$ proveniente de la fijación, tendencia también observada, aunque con menor pendiente, en el tratamiento $S_{3}$ (stress en la fase vegetativa), dejando entrever que los daños sufridos en la tanto en la planta como en su capacidad para ser infectada y formar nuevos nódulos no son irreversibles, lo que concuerda con lo señalado por Rathore et al. (1981), quien indica que especialmente en soya, la falta de agua durante los estadios de formación de nódulos y cerca de la floración no afectó la cantidad de $\mathrm{N}$ asimilado y la producción.

Por otra parte, la inducción de stress durante la floración $\left(S_{4}\right)$ provocó un decremento sostenido del porcentaje de $\mathrm{N}$ proveniente de la atmosfera, indicativo de que la nodulación y la fisiología normal de la simbiosis fueron seriamente afectadas, quedando sin capacidad de regeneración de los nódulos y su actividad.

De acuerdo a la figura 2, el riego durante todo el ciclo de cultivo determinó que cierta proporción del $\mathrm{N}$ asimilado por la planta provenga de la atmósfera, con valores desde $27.66 \%$ hasta $36.47 \%$ y sin variación significativa en todo el ciclo, dentro del rango indicado por Danso (1995), quien afirma que el fréjol común puede suplir entre el 25 y $50 \%$ de su requerimiento.

Se considera que el anterior tratamiento representa las condiciones ideales para el cultivo de fréjol y 
aun así, un gran porcentaje de $\mathrm{N}$ que la planta necesita proviene de la forma combinada presente en el suelo y el $\mathrm{N}$ aportado por el fertilizante solamente cubriría entre el 8 y $10 \%$ de los requerimientos del cultivo.

La figura 3 muestra que el SH durante todo el ciclo de cultivo $\left(S_{2}\right)$ reduce sustancialmente el aporte de $\mathrm{N}$ proveniente de la atmósfera, salvo durante la fase vegetativa en la que se inicia la aplicación del tratamiento, y la desecación del suelo y el agotamiento de la humedad hasta provocar stress hídrico tomaron algún tiempo; esto determinó que durante esta fase la FBN aporte con un significativo porcentaje del $\mathrm{N}$ a la planta, llegando incluso a $39.08 \%$. En el caso de cultivos de secano, es necesaria la programación de los ciclos de cultivo de manera que la siembra y el inicio de la fase más crítica del cultivo, como es la floración, coincidan con las épocas de lluvia de la zona. La producción de fréjol en áreas deficitarias en agua, implica la utilización de una mayor cantidad de fertilizantes nitrogenados, de acuerdo con las recomendaciones del PPI (2010).

El SH provocado durante la fase vegetativa muestra la reducción tanto de la utilización del $\mathrm{N}$ del fertilizante como de la fijación biológica, dependiendo principalmente del nitrógeno combinado presente en el suelo (Marino et al. 2006). También debe considerarse el bajo porcentaje de fijación a que la formación y desarrollo de los nódulos se halla en los estadios iniciales. Cuando el factor limitante es eliminado, el desarrollo de los nódulos y su capacidad de fijación se recuperan e incluso son mejores que los del tratamiento sin stress (Ramos et al. 1999), logrando suplir más del $38 \%$ de sus necesidades de $\mathrm{N}$ por medio de la fijación biológica, siendo el tratamiento $S 3$ el que mayor porcentaje de FBN presentó (Figura 3).

En la Figura 3, se observan también los porcentajes de nitrógeno derivado de la fijación biológica para los tratamientos $S_{4}, S_{5}$ y $S_{6}$, respectivamente. Durante las fases con riego normal la FBN registra porcentajes mayores a los presentes cuando se aplicó el stress hídrico, especialmente en el caso de $S_{4}$ (stress durante la floración) y $S_{5}$ (stress durante el llenado de vaina). La proporción de FBN registrada para la aplicación de stress hídrico en la fase de maduración del grano es mayor que para los restantes tratamiento en todas las fases, indicativo de que la falta de humedad no afecta la FBN en la fase final del cultivo, ya que la mayor parte de las reservas están conformadas y en proceso de maduración, lo cual concuerda con lo explicado por Ramos et al. (1999).

La falta de agua en el suelo durante todo el ciclo de cultivo $\left(S_{2}\right)$, durante la floración $\left(S_{4}\right)$ y durante el llenado de vainas $\left(S_{5}\right)$ afecta drásticamente la FBN en el cultivo de fréjol. La falta de agua en el suelo durante la fase de crecimiento vegetativo $\left(S_{3}\right)$ y la maduración del grano $\left(S_{6}\right)$ no afectan la fijación biológica de nitrógeno, alcanzando valores del 38\%, muy similares al cultivo que no sufrió deficiencia de agua en el suelo durante todo el ciclo $\left(S_{l}\right)$, lo que permitiría ahorrar agua de riego en estas etapa.

La información científica generada permite establecer un manejo racional de agua en las fases críticas de desarrollo del cultivo, especialmente durante la floración. Esto aseguraría un mejor rendimiento del cultivo, afianzando la seguridad alimentaria de la comunidad.

\section{Conflictos de intereses}

Esta investigación se la realizó en la Granja Experimental "La Tola" de la Universidad Central del Ecuador y no presenta conflictos de interés. 


\section{Agradecimientos}

Los autores agradecen a la Universidad Central del Ecuador, Universidad Técnica de Ambato Facultad de Ciencias Agropecuarias y a la Comisión Ecuatoriana de Energía Atómica, por el apoyo técnico, científico y logístico realizado a la presente investigación.

\section{Literatura citada}

Bacchi OOS, Reichardt K, Calvache M. Neutron and gamma probes: Their use in agronomy. Vienna, International Atomic Energy Agency; 2002. p. 75. (Training Course Series 16).

Calvache M, Portezan O, Silva J, Reichardt K. Conductividade hidráulica de um solo Typic Haplustoll em condições de campo. In: Congresso Brasileiro Da Ciência Do Solo, 25., Viçosa, 1995. Resumos Expandidos. Viçosa: SBCS; 1995. p. 63-5.

Calvache M, Reichardt K, Bacchi OOS, DouradoNeto D. Deficit irrigation at different growth stages of the common bean (Phaseolus vulgaris L., cv. Imbabello). Sci Agric 1997;54(NE): $1-16$.

Calvache M. Riego andino tecnificado. Universidad Central del Ecuador; 2013. p. 296.

Campbell SG, Stokle CO. Prediction and simulation of water use in agricultural systems. In International Crop Science I, based on the International Crop Science Congress sponsored by Iowa State University and the Crop Science Society of America, Ames, Iowa 14-22 July; 1993. p. 67-74.

Castellanos JZ, Pena-Cabrales JJ, Acosta-Gallegos JA. ${ }^{15} \mathrm{~N}$-determined dinitrogen fixation capaci105

$$
\text { ty of common bean (Phaseolus vulgaris) culti- }
$$

vars under water stress. J Agric Sci 1996; 126:327-33.

Corporación de Ppromoción de Eexportaciones e iInversiones CORPEI. 2009. Perfiles de productos. Perfil de fréjol. Coordinadora del Ccentro de Informacióninformación e iInteligencia Ccomercial-CICO de CORPEI. Ecuador; 2009. p. 27.

Danso SKA. Sustainable agriculture: The role of biological nitrogen fixing plants. In Nuclear Techniques in Soil-Plant studies for sustainable agriculture and environment preservation. Proceedings of a Symposium, Viena, 17-21 October; 1995. FAO IAEA. p. 735.

Duncan D B.Multiple range and multiple $F$ tests. Biometrics 1955;11:1-42.

Faust H, Sebastianelli JA, Axmann H. Manual de laboratorio; métodos para el análisis de $15 \mathrm{~N}$. Curso interregional de entrenamiento sobre el uso de $15 \mathrm{~N}$ en ciencias de suelos, nutrición vegetal y biotecnología agrícola. FAO/OIEA, Leipzig, Alemania; 1987. p. 30-45.

Fereres E, Orgaz F, Villalobos FJ. Water use efficiency in sustainable agricultural systems. In International Crop Science I, based on the International Crop Science Congress sponsored by Iowa State University and the Crop Science Society of America, Ames, Iowa 14-22 July; 1992. p. 83-9.

Hardarson G Methods for enhancing symbiotic nitrogen fixation. Plant Soil 1993;152(1):1-17.

Hardarson G, Danso SKA, Zapata F Dinitrogen fixation measurements in alfalfa-ryegrass swards using nitrogen-15 and influence of the reference crop. Crop Sci 1988;28:101-5.

Hardarson G, Zapata F, Danso SKA. Dinitrogen fixation measurements in Alfalfa-Ryegrass 
Swards Using Nitrogen-15 and Influence of the Reference Crop. Crop Sci 1988;28(1):101-5.

Hardarson, G. 1990. Empleo de las técnicas nucleares en los estudios de la relación suelo-planta. Viena, Organismo Internacional de Energía Atómica; 1990. p. 173.

Hungria M, Vargas MA. Environmental factors affecting $\mathrm{N}_{2}$ fixation in grain legumes in the tropics, with an emphasis on Brazil. Field Crops Res 2000;65(2):151-64.

Instituto Nacional de Meteorología e Hidrología INAHMI. 1996. Valores promedio de 16 años de la Estación Meteorológica "La Tola". Anuarios meteorológicos 1980-1996.

López-Guerrero MG, Ormeño-Orrillo E, Velázquez E, Rogel MA, Acosta JL, Gónzalez V, et al. Rhizobium etli taxonomy revised with novel genomic data and analyses. Syst Appl Microbiol 2012;35(6):353-8.

Marino D, González EM, Arrese-Igor C. Drought effects on carbon and nitrogen metabolism of pea nodules can be mimicked by paraquat: evidence for the occurrence of two regulation pathways under oxidative stresses. J Exp Bot 2006;57(3):665-73.

Peralta E, Murillo A, Caicedo C, Pinzón J, Rivera M. S.f. Manual Agrícola de Leguminosas; cultivo y costos de producción. INIAP, PROFIRIZA, Universidad de Minnesota; COSUDE; p. 49.

Potash and Phosphate Institute PPI. Manual de fertilidad de los suelos. Atlanta, Georgia, USA, PPI; 1988. p. 85.
Rai R, Dash PK, Mohapatra T, Singh A. Phenotypic and molecular characterization of indigenous rhizobia nodulating chickpea in India. Indian $\mathbf{J}$ Exp Biol 2012;50(5):340-50.

Ramos MLG, Gordon AJ, Minchin FR, Sprent JI, Parsons R. Effect of water stress on nodule physiology and biochemistry of a drought tolerant cultivar of common bean (Phaseolus vulgaris L.). Ann Bot 1999;83:57-63.

Rathore T, Chonkar PK, Sanchan R, Ghidyal B.Effect of soil moisture stress on legumerhizobium symbiosis in soybeans. Plant Soil 1981;60(3):445-50.

Ribeiro RA, Ormeno-Orrillo E, Dall'Agnol RF, Graham PH, Martinez-Romero E, Hungria M. Novel Rhizobium lineagesisolated from root nodules of the common bean (Phaseolusvulgaris L.) in Anden and Mesoamerican areas. Res Microbiol 2013;164(7):740-8.

Murray JD. Invasion by invitation: rhizobial infection in legumes. Mol Plant Microbe Interact 2011;24(6):631-9.

Singh JS, Pandey VC, Singh DP. Efficient soil microorganisms: a new dimension for sustainable agriculture and environmental development. Agric Ecosyst Environ 2011;140(3-4):339-53.

Tikhonovich IA, Provorov NA. Microbiology is the basis of sustainable agriculture: an opinion. Ann Appl Biol 2011;159(2):155-68.

Wilkins M. Advanced Plant Physiology. Third imp. England. Longman; 1987. p. 514. 